\title{
Lymphocytic colitis complicated by a mass in the terminal ileum
}

Chee-Kin $\underline{\mathrm{Hui}}^{1,2}$, MD

\begin{abstract}
Lymphocytic colitis is a chronic inflammatory disease affecting the bowel. The clinical course of lymphocytic colitis is believed to be benign with watery diarrhoea. We report herein what is, to the best of our knowledge, the first case of lymphocytic colitis complicated by a terminal ileal mass. A 23-year-old man presented with diarrhoea. Blind biopsies of samples taken from the terminal ileum, caecum and ascending colon showed features of lymphocytic colitis. He declined treatment with budesonide or 5-aminosalicylates. He presented 14 months later with pain over the right lumbar region and nausea. Computed tomographic enteroclysis showed a focal soft tissue enhancing mass at the terminal ileum. Excision of the soft tissue mass revealed that it was reactive nodular lymphoid hyperplasia with fibrous granulation tissue. In conclusion, an untreated lymphocytic colitis may result in the formation of an inflammatory mass lesion.
\end{abstract}

Keywords: abdominal pain, computed tomographic enteroclysis, lymphocytic colitis, nausea, terminal ileal mass

\section{INTRODUCTION}

Lymphocytic colitis is a chronic inflammatory disease affecting the bowel. ${ }^{(1)}$ It can occur at any age, with a peak incidence at around 60 years old. The annual incidence of lymphocytic colitis is 4.4 per 100,000 women and 3.0 per 100,000 men. ${ }^{(2-4)}$ We herein report a case of lymphocytic colitis that was complicated by a terminal ileal mass, resulting in persistent abdominal pain and nausea. To the best of our knowledge, this is the first such case reported.

\section{CASE REPORT}

A 23-year-old man presented with a year-long history of episodic diarrhoea that occurred about once every two to three months and would last for two to four weeks. There was no associated abdominal pain, weight loss, decreased appetite, fever or malaise. A physical examination was conducted. Tests were also performed to find out the patient's complete blood picture, erythrocyte sedimentation rate, C-reactive protein level, renal biochemistry, liver biochemistry, coeliac disease antibodies, autoimmune markers and thyroid function. The results were all unremarkable.

Results of a colonoscopy up to the terminal ileum were also normal. Blind biopsies of samples taken from the terminal ileum, caecum and ascending colon showed mixed chronic inflammatory cell infiltrate in the lamina propria consisting of lymphocytes and plasma cells. There were scattered eosinophils with increased intraepithelial lymphocytes at $\geq 20$ per 100 surface epithelial cells (Fig. 1). There was no thickening of the basement membrane or focal deposition of collagen.

On the other hand, blind biopsies of samples taken from the transverse colon, descending colon, sigmoid colon and rectum only showed nonspecific inflammation. Overall, the histological features were suggestive of lymphocytic colitis involving the

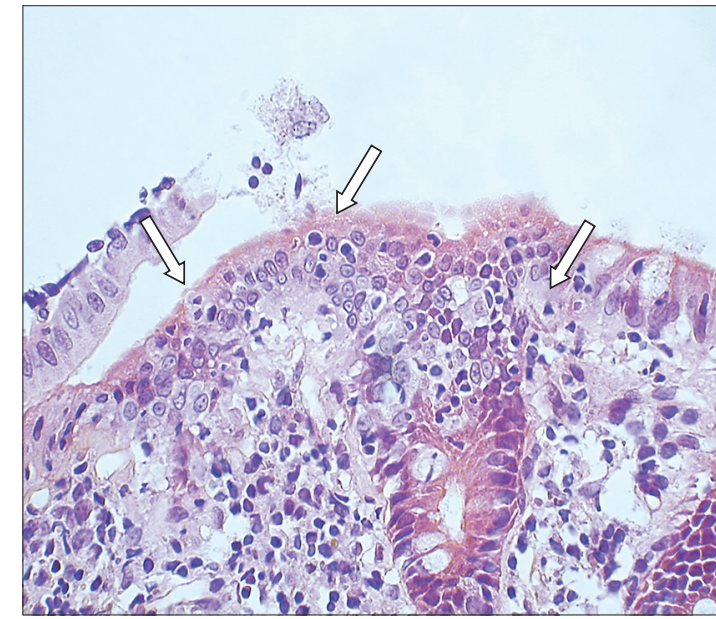

Fig. 1 Photomicrograph of a colonic biopsy shows increased intraepithelial lymphocytes (arrows) in the surface epithelium (Haematoxylin \& eosin, $\times 400)$.

terminal ileum, caecum and ascending colon. A thorough search for risk factors associated with lymphocytic colitis such as drugs, autoimmune disorder, thyroid disorder and bacterial infection were all negative. The patient declined budesonide or 5-aminosalicylates and was treated with an antispasmodic, antidiarrhoea agent on an as-needed basis. He continued to have episodic diarrhoea that would spontaneously subside. It did not affect his daily activities.

The patient presented 14 months after the colonoscopy with an insidious onset of pain over the right lumbar region and nausea. The pain and nausea would occur 2-3 hours after every meal. There was no vomiting, headache, pigmentation or rash. He had not had diarrhoea for two months. Results of a physical examination at this time were unremarkable. Test results of his electrolytes, cortisol, fasting sugar, complete blood picture, erythrocyte sedimentation rate, C-reactive 

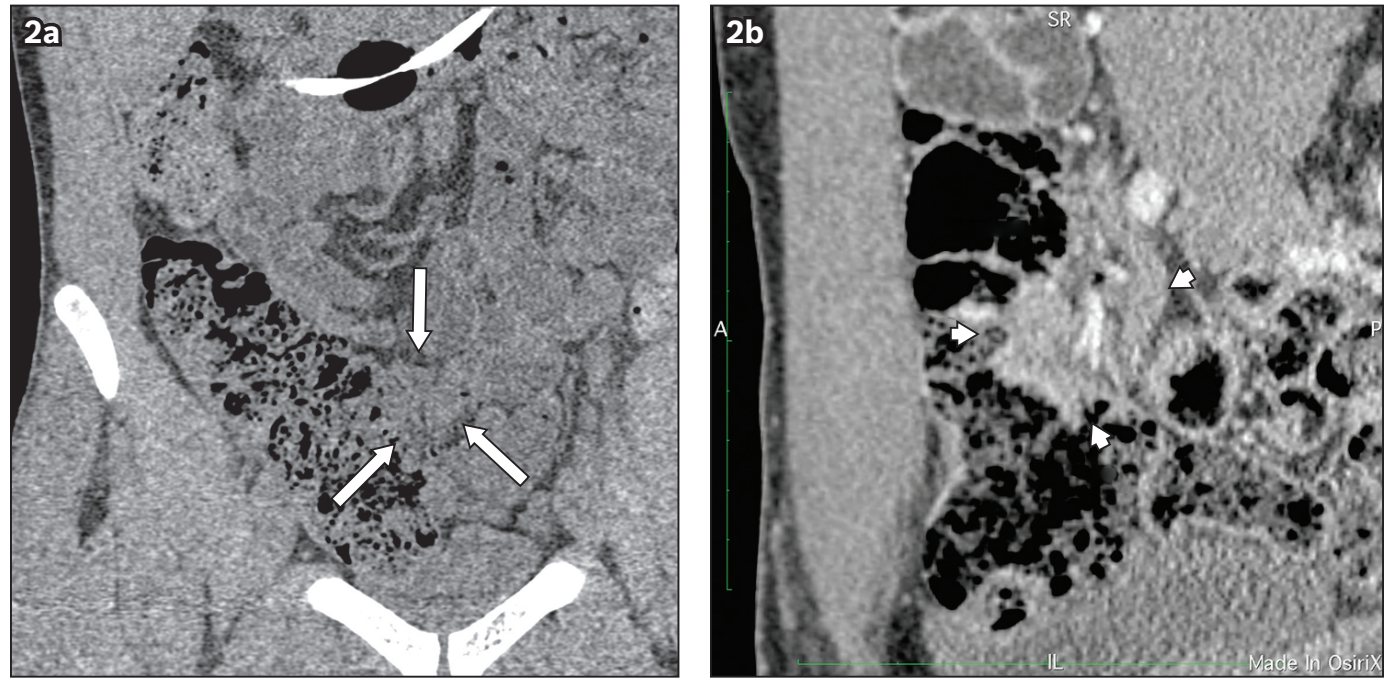

Fig. 2 CT enteroclysis images show the coronal view of the terminal ileal mass with indentation and displacement of ileum (arrows) in (a) noncontrast; and (b) contrast views.
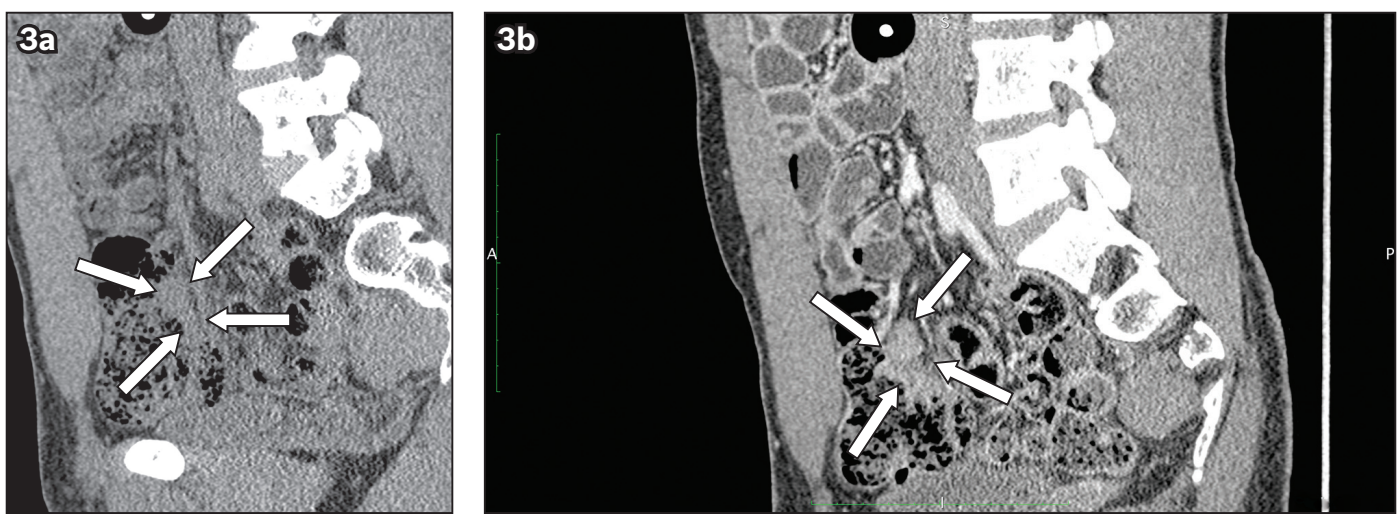

Fig. 3 CT enteroclysis images show the sagittal view of the terminal ileal mass with indentation and displacement of ileum (arrows) in (a) noncontrast; and (b) contrast views.

protein level, renal biochemistry, liver biochemistry, amylase, autoimmune markers, complement factors, immunoglobulins, coeliac disease antibodies and Yersinia enterocolitica antibodies, and findings from a routine microscopy urinalysis were all unremarkable. An upper endoscopy yielded results that were normal down to the second part of the duodenum. Multiple biopsies taken from the second part of the duodenum and stomach were all unremarkable without any evidence of coeliac disease. Ultrasonography of the upper abdomen and computed tomography (CT) of the whole abdomen were both unremarkable.

Over a month's time, the patient lost $7 \mathrm{~kg}$ of weight and the abdominal pain and nausea after every meal became increasingly severe. However, his diarrhoea did not recur. CT enteroclysis was subsequently performed. It showed a focal soft tissue enhancing mass at the terminal ileum that was $2.2 \mathrm{~cm} \times 3.1 \mathrm{~cm} \times 3.1 \mathrm{~cm}$ (Figs. 2a \& b). Mass effect with indentation and displacement of the terminal ileum was observed (Figs. 3a \& b). Colonoscopy was repeated up to the terminal ileum and advanced to $15 \mathrm{~cm}$ beyond the ileocaecal valve. Multiple lymphoid follicles were seen at the terminal ileum. Furthermore, multiple biopsies taken from the terminal ileum showed mild nonspecific mixed inflammatory cell infiltrate in the lamina propria. There was no evidence of viral inclusion, granulomatous inflammation, parasites, villous atrophy, dysplasia or malignancy. Biopsies taken from the caecum and ascending colon showed a slight increase in intraepithelial lymphocytes ( $>5$ and $<20$ per 100 surface epithelial cells).

In view of the inconclusive findings of terminal ileal biopsies and the patient's persistent symptoms, laparotomy with excision of the terminal ileal soft tissue mass was performed. The ileal specimen showed good preservation of the overall architecture. In areas of the mucosa, there was dense lymphoid infiltrate accompanied by scattered reactive lymphoid follicles. The infiltrate was predominantly composed of small lymphoid cells mixed with plasma cells. The submucosal tissue showed fibrosis (fibrous) and granulation tissue. On immunostaining, there were nodular aggregates of CD20+ B cells separated by zones of CD3+ T cells. The follicle centres were CD10+. The B cells did not show co-expression of CD5 or cyclin D1. Therefore, the overall features were those of nodular lymphoid hyperplasia, especially since the lower border was discrete and at the plane of the muscularis mucosae.

Although the patient's abdominal pain and nausea subsided after surgery, he was commenced on $9 \mathrm{mg}$ of budesonide daily. The dose of budesonide was slowly tapered off over a 32-week 
period and he remained asymptomatic 52 weeks after it was discontinued.

\section{DISCUSSION}

Lymphocytic colitis and collagenous colitis are considered two distinct histological subtypes of microscopic colitis. However, as they possess overlapping features, some investigators believe that they are two histological subtypes of the same disease and are clinical manifestations of a spectrum of conditions. ${ }^{(4)}$ Nyhlin et al estimated that lymphocytic colitis and collagenous colitis account for $20 \%$ of cases of chronic non-bloody diarrhoea. ${ }^{(5)}$

Lymphocytic colitis is believed to follow a benign clinical course and present as watery diarrhoea. It is not associated with blood loss. The majority of patients grow accustomed to diarrhoea symptoms. Although such diarrhoea can be severe, it is not known to be associated with deterioration in the clinical condition or changes in electrolyte levels. ${ }^{(2-4)}$ Instead, the disease waxes and wanes. A high spontaneous remission rate of $93 \%$ has been reported. ${ }^{(6)}$

The only complications that have been reported to occur in cases of microscopic colitis are spontaneous colonic perforations or colonoscopy-induced perforations. These complications are caused by the presence of mucosal tears. ${ }^{(7)}$ However, spontaneous or colonoscopy-induced colonic perforations complicating microscopic colitis have mostly been reported in cases of collagenous colitis, rather than in lymphocytic colitis. ${ }^{(7-9)}$ In view of the high rate of spontaneous remission and lack of complication, it was not difficult to understand why the patient declined treatment with 5-aminosalicylate and budesonide.

When the terminal ileal mass was discovered in our patient, it was not suspected to be associated with lymphocytic colitis. This is because the common pathological conditions expected to be found in the terminal ileum are tuberculosis, small bowel lymphoma, Yersinia infection, neuroendocrine tumour or inflammatory bowel disease. ${ }^{(10)}$ As the investigation failed to yield a diagnosis and the patient continued to experience persistent 'obstructive' symptoms, the mass was completely excised.

The mass could have been formed by repeated inflammation in the terminal ileum due to the lymphocytic colitis. In a similar case, Padmanabhan et al demonstrated that intraepithelial lymphocytosis and inflammation can occur in the terminal ileum of patients with lymphocytic colitis. In fact, they showed that the terminal ileum may be involved in up to $78 \%$ of patients with lymphocytic colitis. This involvement may be a continuation of the disease extending into the terminal ileum, similar to the 'backwash ileitis' observed in ulcerative colitis. ${ }^{(11)}$ On the other hand, it may indicate that the small bowel may have a role in the pathogenesis of lymphocytic colitis. The repeated inflammation in the terminal ileum over the period of 14 months (as the patient declined treatment with either budesonide or 5-aminosalicylates) may have resulted in the reactive nodular lymphoid hyperplasia.

This repeated inflammation, which waxed and waned, may have led to the formation of fibrous and granulation tissues. The mass was then formed from the fibrosis (fibrous) and granulation tissue. The indentation and displacement of the terminal ileum caused by the mass resulted in the patient's diarrhoea symptom, which would occur about 2-3 hours after a meal. Intraepithelial lymphocytes were not found in the terminal ileum section, as the inflammation may have already 'burnt out' at the time of surgery.

The reduction in the number of intraepithelial lymphocytes found in the caecum and ascending colon on a repeat colonoscopy, compared with the number found at the time of diagnosis, was taken as further evidence that the lymphocytic colitis had waned. However, the intraepithelial lymphocytes found in the caecum and ascending colon on the repeat colonoscopy was $>20$ intraepithelial lymphocytes per 100 surface epithelial cells, which was still higher than the number regarded as normal (i.e. $>5$ intraepithelial lymphocytes per 100 surface epithelial cells). . $3,4,6,12)$ Bjørnbak et al have found that some patients with so-called "incomplete findings of microscopic colitis" do not meet the pathological criteria for microscopic colitis but have developed the condition. ${ }^{(12)}$

One can only speculate whether the inflammatory mass would have responded to a course of treatment with budesonide, thereby sparing the patient from surgery. Budesonide is now the drug of choice for the treatment of lymphocytic colitis. ${ }^{(3,4,6)}$ This is because of its good side-effect profile; budesonide has limited systemic availability due to its extensive first-pass metabolism by cytochrome P450 enzymes. ${ }^{(4,6)}$

In conclusion, lymphocytic colitis may not be as benign as was believed. Untreated lymphocytic colitis may result in repeated inflammation that waxes and wanes. This inflammation may in turn result in the formation of an inflammatory mass lesion. More studies on the natural history of lymphocytic colitis are needed in order to determine its outcome, prognosis and progression, if any.

\section{ACKNOWLEDGEMENTS}

I would like to thank Dr Chan Kui Fai, Quality Healthcare Diagnostic and Imaging Centre, Kowloon, Hong Kong, for the CT images; and Dr Ah Chong Ah Kian, Pedder Clinic and Owens, Trodd and Partners, Hong Kong, for the surgical care.

\section{REFERENCES}

1. Pardi SP, Kelly CP. Microscopic colitis. Gastroenterology 2011; 140:1155-65.

2. Temmerman F, Baert F. Collagenous and lymphocytic colitis: systematic review and update of the literature. Dig Dis 2009; 27 Suppl 1:137-45.

3. Brown WR, Tayal S. Microscopic colitis. A review. J Dig Dis 2013; 14:277-281.

4. Rasmussen MA, Munck LK. Systematic review: are lymphocytic colitis and collagenous colitis two subtypes of the same disease - microscopic colitis? Aliment Pharmacol Ther 2012; 36:79-90.

5. Nyhlin N, Bohr J, Eriksson S, Tysk C. Systematic review: microscopic colitis. Aliment Pharmacol Ther 2006; 23:1525-34

6. El-Salhy M, Gundersen D, Hatlebakk JG, Hausken T. Clinical presentation, diagnosis, pathogenesis and treatment options for lymphocytic colitis (Review). Int J Mol Med 2013; 32:263-70.

7. Allende DS, Taylor SL, Bronner MP. Colonic perforation as a complication of collagenous colitis in a series of 12 patients. Am J Gastroenterol 2008; 103:2598-604.

8. Freeman HJ. Complications of collagenous colitis. World J Gastroenterol 2008; 14:1643-5.

9. Hussain Z, Kelly S, Clarke A, Adams S, Miller G. Colonic perforation 
in collagenous colitis: a systematic review of a rare complication and guidance on management. Surg Endosc 2010; 24:2930-4.

10. Hui CK. Celiac disease presenting as idiopathic terminal ileum obstruction. Journal of Gastroenterology and Hepatology Research 2013; 2:652-4.

11. Padmanabhan V, Callas PW, Li SC, Trainer TD. Histopathological features of the terminal ileum in lymphocytic and collagenous colitis: a study of 32 cases and review of the literature. Mod Pathol 2003; 16:115-9.

12. Bjørnbak C, Engel PJ, Nielsen PL, Munck LK. Microscopic colitis: clinical findings, topography and persistence of histopathological subgroups. Aliment Pharmacol Ther 2011; 34:1225-34. 